\title{
Immune response and distribution of antigen in chickens after infection LPAIV (H4N6)
}

\author{
Denys Muzyka*1, Hyun Lillehoj ${ }^{2}$, Olexandr Rula ${ }^{1}$ and Borys Stegniy ${ }^{1}$ \\ 'Laboratory of Avian Diseases Epizoothology, National Scientific Center Institute of Experimental and Clinical Veterinary Medicine, \\ Kharkiv, Ukraine; ${ }^{2}$ Animal Parasitic Diseases Laboratory, Beltsville, MD, USA
}

\section{Objective}

To study the immune response in chicken on the administration of LPAIV isolated from the natural reservoir.

\section{Introduction}

Influenza is a serious problem for the health of people, animals and birds. Therefore, comprehensive study of influenza virus, its natural reservoir, pathogenesis and immune response will provide further opportunity to ensure protection for animals, birds and people from this infection.

\section{Methods}

Four-week-old commercial chickens were intranasally inoculated with a H4N6 LPAIV A/Garganey/Chervonooskilske/4-11/2009 (H4N6), isolated from the cloacal swab of clinically healthy garganey in 2009 in Ukraine. Cecum, spleen, lung, and trachea samples were collected from infected chickens on $1-14$ dpi and examined by immunohistochemical and virology techniques. On these days, we collected blood samples for serological analysis. Detection of antibodies to avian influenza virus subtype H4 was performed with chicken serum samples by HI test and ELISA. The studies were done according to IACUC.

\section{Results}

Upon intravenous and intranasal infection with this virus (A/Garganey/Chervonooskilske/4-11/2009), no clinical signs were observed in chickens and no pathological changes were found at necropsy. Infection of poultry with this virus provoked an antibody response at 10 days after intranasal inoculation which ranged from $1: 8$ to $1: 32$ serum antibody titers. Only 2 of 5 chickens were positive by the HI test and 3 of 5 were positive by ELISA at intranasal inoculation. All 10 chickens were positive both by HI test and by ELISA after intravenous inoculation. Specific antibodies (HI test) to influenza virus $\mathrm{H} 4$ were detected in titer ranges of 1:128 to 1:1024.

In immunohistological studies, the respiratory tract organs (lungs and trachea) showed higher level of humoral immunity (IgM, IgG, IgA-expressing cells) in the lung compared to the trachea. Also, indicators of cell mediated immunity as measured by the CD4 and macrophage markers were higher in LPAIV-infected chickens in the lungs at 14 days post infection compared to uninfected chickens. Lymphocytes expressing CD8 were increased starting $7 \mathrm{dpi}$. The chickens in the infected group showed 2 times higher levels of CD8 cells compared to the control chickens. IFN- $\gamma$ transcripts were observed in the AI-infected chickens starting at 7dpi that coincides with the increasing level of

CD4 cells. The number of lymphocytes which secrete IL-2 and IL-15 in AI-infected chickens were in general 1.5 to 2 times higher compared to the uninfected chickens. In AIV-infected chickens, the level of cells expressing IFN- $\gamma$, IL-2, and IL-15 increased at 7-days after infection. The peak time coincided with a period of increasing CD8 cells. However, there was no significant difference in these cytokine levels between the AIV-infected and uninfected groups.

In the cecum, lower levels of CD4 cells were seen on 5 dpi but levels slowly increased from $7 \mathrm{dpi}$ to $14 \mathrm{dpi}$ following AIV infection.
In the ceca, a significant increase in the number of cells expressing IgM and IgG was found.

LPAIV infection induced an increase in macrophages and lymphocytes expressing CD4 and CD8 in the spleen throughout the period examined in this study indicating their role in host response to viral infection. The levels of macrophages in chickens of AIV infected group were 2 times higher than the control after $1 \mathrm{dpi}$.

\section{Conclusions}

Although infection with a LPAIV did not cause obvious clinical disease, viral replication was detected in the trachea and spleen and both local and systemic cellular and humoral immune responses were elicited in these LPAIV-infected chickens. Our results indicate the potential possibility for infection of poultry with viruses isolated from wild birds. But currently it is not completely known why some viruses from wild birds can cause infection in poultry, while others can not. Further study of the immune response will enable us to determine the features of the pathogenesis of low pathogenic avian influenza.

\section{Keywords}

avian influenza; immune response; chicken

\section{Acknowledgments}

Our research was funded by USDA projects P382a and P444 through the Ukrainian Science and Technology Center. The authors express special gratitude Dr. Pavlo Shutchenko.

\section{*Denys Muzyka}

E-mail: dmuzyka77@gmail.com 